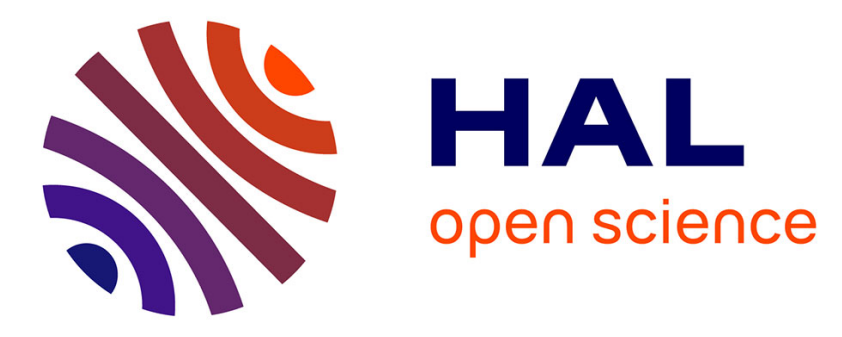

\title{
Mechanical model of giant photoexpansion in a chalcogenide glass and the role of photofluidity
}

Manuel Buisson, Yann Gueguen, Romain Laniel, Christopher Cantoni, Patrick Houizot, Bruno Bureau, Jean-Christophe Sangleboeuf, Pierre Lucas

\section{- To cite this version:}

Manuel Buisson, Yann Gueguen, Romain Laniel, Christopher Cantoni, Patrick Houizot, et al.. Mechanical model of giant photoexpansion in a chalcogenide glass and the role of photofluidity. Physica B: Condensed Matter, 2017, 516, pp.85-91. 10.1016/j.physb.2017.05.001 • hal-01518188

\author{
HAL Id: hal-01518188 \\ https://hal.science/hal-01518188
}

Submitted on 13 Jul 2017

HAL is a multi-disciplinary open access archive for the deposit and dissemination of scientific research documents, whether they are published or not. The documents may come from teaching and research institutions in France or abroad, or from public or private research centers.
L'archive ouverte pluridisciplinaire HAL, est destinée au dépôt et à la diffusion de documents scientifiques de niveau recherche, publiés ou non, émanant des établissements d'enseignement et de recherche français ou étrangers, des laboratoires publics ou privés. 


\title{
Mechanical model of giant photoexpansion in a chalcogenide glass and the role of photofluidity
}

\author{
Manuel Buisson ${ }^{1}$, Yann Gueguen ${ }^{1}$, Romain Laniel ${ }^{1}$, Christopher Cantoni ${ }^{2}$, Patrick \\ Houizot $^{1}$, Bruno Bureau ${ }^{3}$, Jean-Christophe Sangleboeuf ${ }^{1}$, and Pierre Lucas ${ }^{2, *}$ \\ ${ }^{1}$ Department Mechanics \& Glasses, IPR UMR UR1-CNRS 6251, Universite de Rennes 1, Campus de Beaulieu, \\ Rennes Cedex, 35042, France \\ ${ }^{2}$ Department of Materials Science and Engineering, University of Arizona, Tucson, Arizona 85712, USA \\ ${ }^{3}$ Equipe Verres et Ceramiques, UMR 6226 Sciences Chimiques de Rennes, Universite de Rennes 1, Glass and \\ Ceramics Laboratory, Campus de Beaulieu, Avenue du General Leclerc 35042, Rennes Cedex, France
}

\begin{abstract}
.
An analytical model is developed to describe the phenomenon of giant photoexpansion in chalcogenide glasses. The proposed micro-mechanical model is based on the description of photoexpansion as a new type of eigenstrain, i.e. a deformation analogous to thermal expansion induced without external forces. In this framework, it is the viscoelastic flow induced by photofluidity which enable the conversion of the self-equilibrated stress into giant photoexpansion. This simple approach yields good fits to experimental data and demonstrates, for the first time, that the photoinduced viscous flow actually enhances the giant photoexpansion or the giant photocontraction as it has been suggested in the literature. Moreover, it highlights that the shear relaxation time due to photofluidity controls the expansion kinetic. This model is the first step towards describing giant photoexpansion from the point of view of mechanics and it provides the framework for investigating this phenomenon via numerical simulations.
\end{abstract}

Keywords: Photoexpansion, photofluidity, photoinduced fluidity, chalcogenide glasses, eigenstrain

*Corresponding author: pierre@u.arizona.edu; Tel: 15203222311

\section{INTRODUCTION}

Chalcogenide glasses cover a wide range of applications [1,2]; first and foremost because of their transparency in the infrared range. This includes thermal imaging cameras [3], generation of new infrared sources [4], chemical and biological sensors [5], optical data storage [6], etc. Chalcogenide glasses are also photosensitive materials. They exhibit a wide range of photoinduced phenomena [7, 8] including: photodarkening/bleaching[9], photoinduced fluidity $[10,11]$, photoinduced structural relaxation $[12,13]$, photoinduced expansion [14] or contraction 
[15], etc. Some of these effects can be scalar or vectorial, i.e.: isotropic or anisotropic respectively, depending on the light polarization [16].

Most photostructural changes can be induced by irradiation with either above or below bandgap light. Some photoinduced effects are much more pronounced in the sub-bandgap range [17], including giant photoexpansion-contraction [14, 15], photofluidity $[10,11]$ and photoinduced structural relaxation [18], because, sub-bandgap light permits to illuminate a larger volume. In addition, above bandgap irradiation is associated with high absorption that is sufficient to induce a significant warming and even potential damage, while sub-bandgap irradiation has a negligible thermal contribution under standard irradiation power. Several proofs of the optical origin and athermal nature of photoinduced effects exist including the fact that photofluidity is more pronounced at low temperature [10] and exhibits polarization effects [19] or the formation of wavelength specific Bragg gratings [20]. Nevertheless, it has been recently suggested that the thermal contribution to the photoexpansion itself may not be negligible even for weakly absorbed light [21].

Giant photoexpansion in chalcogenide glasses has many applications including the production of adaptive lenses [22], microlenses arrays [23], optical fiber microlenses [24], structured concave/convex lenses [15] and relief patterning for the production of gratings [19, 25, 26]. This technique is promising, since complex optical elements can be optically created in a single step. Nevertheless, if the kinetic and the geometry of the expansion cannot be predicted, the development of this technique will be limited because of the large number of tests required to produce the desired lens geometry. Hence a mechanical model describing the photoexpansion would considerably improve the predictability of the expansion geometry and would generally ease the development of photo induced relief patterning techniques. However, the giant photoexpansion is a phenomenon which is very difficult to model since its physical origin is still debated, and since other photoinduced effects may contribute to the expansion. Usually, bulk glasses or thin films are irradiated with a laser beam having a diameter much lower than the sample size. This way, the irradiated volume fluidifies and expands while being confined by the quasi-rigid volume surrounding the laser path which remains mostly unchanged. If the irradiated volume can expand easily in the direction of free surfaces, it is stressed in the direction perpendicular to the bean axis: this is where the viscoelastic properties of the glass come into play. This can be easily understood thanks to the phenomenological model proposed by Tanaka et al. [27]. Despite clear indications that the phenomenon is governed by mechanical stress, the mechanical aspect of this phenomenon has been largely ignored or poorly treated. Various models of photoexpansion have been proposed in the literature [17, 28], but they all treat the expansion as if it was stress-free. The objective of the present work is to treat the photoexpansion from the point of view of mechanics, by taking into account the combined effect of these stresses and the photoinduced fluidity. We show here, using some simple hypothesis, that the contribution of photofluidity can be treated in a systematic way and that the resulting model gives a good estimation of the kinetic and the strain field of the giant photoexpansion observed experimentally. 


\section{MICRO-MECHANICAL MODEL OF PHOTOEXPANSION VIA A SIMPLE THERMODYNAMICAL APPROACH}

\subsection{Eigen Strain}

Taking into account the physics of the photoexpansion, we assume that viscosity and photoexpansion phenomena induce respectively two so called "eigenstrains": the viscous-strain, $\epsilon^{\mathrm{v}}$, and the photoexpansion strain, $\epsilon^{\mathrm{p}}$. As suggested by Mura [29], eigenstrain refers to such a category of nonelastic strains such as thermal expansion or phase transitions which can induce a self-equilibrated stress field called "eigenstress fields" without any external force and surface constraint. We will not deal with the structural origin of the eigenstrain due to photoexpansion, but only with the resulting internal stress fields.

\subsection{Model basis}

\subsubsection{Athermal nature}

The photoexpansion is assumed to proceed continuously and quasi-statically under permanent irradiation; the kinetic of this evolution is described by a single time parameter $\left(\tau_{\mathrm{A} 0}\right)$. The eigenstrain saturates at long time, and this saturation level is taken to be proportional to the local light intensity. In this work, we will neglect the thermal aspect of the photoexpansion and we will not sort out the question of the physical origin of the corresponding eigenstrain. In fact, we will show here quantitatively that thermo-viscoelastic coupling and heat conduction can be disregarded, as a first approximation. We also assume that photoexpansion occurs in a viscoelastic medium. Indeed, under irradiation, chalcogenide glasses undergo a large and athermal viscosity decrease named photofluidity or photoinduced fluidity [11]. The viscosity under irradiation is inversely proportional to the number of photons absorbed per second per atom [30, 31] and does not depend on the stress [32]: this further justifies the assumption that irradiated glasses undergo a linear viscoelastic behavior. The glass structure is also assumed to be isotropic. For elasticity, the values of the bulk modulus $k$ and of the Poisson's coefficient $v$ given from [33]:

$$
\mathrm{k}=10.2 \pm 0.2 \mathrm{GPa} \quad v=0.307 \pm 0.01
$$

will be adopted for this study.

\subsubsection{Self-focusing}

Since the glass undergoes photoinduced refractive index changes, the glass sample may focus the laser beam along the thickness through a photoinduced self-focusing process [14]. This effect will be neglected in the present model. It was shown that the self-focusing is prominent when the ratio sample thickness/beam radius is much larger that 10 (ref.[14]). In our experiment, this ratio is 9.3. The divergence of the laser beam due to the photoexpansion will also be neglected. Indeed, during the expansion, the average divergence of the beam is lower than $2^{\circ}$. 


\subsection{Model approach}

A detailed mathematical description of the proposed model is presented in the Supplemental Material section. Here we describe the general approach adopted to quantify the mechanical response of the glass under irradiation. First the free energy per unit volume is expressed within the framework of the Generalized Standard Model [34] using two distinct tensors for photoexpansion and viscous flow which are treated as uncoupled. Dissipation due to viscous strain rate is also accounted for using a dissipation potential. These yield a state law for the stress which provides the stress-strain response under irradiation. Photoexpansion has only spherical contribution to straining and viscosity plays a deviatoric role, i.e. only shear viscosity comes into play. The problem is treated as rotationally symmetrical around the z-axis of the beam.

\section{EXPERIMENTAL}

\subsection{Glass synthesis}

The giant photoexpansion has been investigated on $\mathrm{GeSe}_{9}$ glasses. The glass production procedure is detailed in ref.[35]. A glass rod was sliced and cut to the desired testing specimen geometry using a diamond saw. The surfaces of the specimens were mirror polished using $\mathrm{SiC}$ paper and alumina suspension with $0.25 \mu \mathrm{m}$ particle size. The sample thickness was $1.5 \pm 0.02$ $\mathrm{mm}$. The glass transition temperature of $\mathrm{GeSe}_{9}$ is $92^{\circ} \mathrm{C}$ (ref. [30])

\subsection{Irradiation}

A tunable Ti-sapphire laser 3900S from Spectra Physics was used in continuous mode for all the experiments. The Ti-sapphire was pumped with a $5 \mathrm{~W}, 532 \mathrm{~nm}$ laser. The Ti-sapphire laser was tuned to $790 \mathrm{~nm}$ so that the excitation wavelength corresponds to the Urbach edge of the $\mathrm{GeSe}_{9}$ sample. The photon energy is $1.57 \mathrm{eV}$, far below the bandgap $\mathrm{Eg}_{\mathrm{g}}=1.95 \mathrm{eV}$ (ref. [30]) measured as the photon energy corresponding to an absorption of $1000 \mathrm{~cm}^{-1}$. At this photon energy, the absorption coefficient is lower than $10 \mathrm{~cm}^{-1}$ even if the photodarkening is taken into account [30]. The collimated laser beam was perpendicular to the surface and was not focused on the sample. The samples were irradiated for various periods of time, and the profile of the permanent photoexpansion was collected using a stylus profilometer Dektak 6M with a $12.5 \mu \mathrm{m}$ radius diamond tip a few minutes after the irradiation periods. The samples were glued on a metallic support with a hole in the middle, so that no reflection of the laser beam occurred on the metallic support. The power of the laser beam was set at $\mathrm{P}=33.8 \mathrm{~mW}$ using 2.0 and 0.2 neutral density filters with a rotationally symmetrical intensity profile. The laser beam profile was characterized and is plotted on Figure 1. This experimental profile is modeled by a Gaussian fit according to equation (2).

$$
I(r)=I_{0} \exp \left[-\frac{2 \cdot r^{2}}{R^{2}}\right]
$$

where $\mathrm{I}_{0}=2 \mathrm{P} / \pi \mathrm{R} 2=82.45 \mathrm{~W} \cdot \mathrm{cm}^{-2}$ is the maximum intensity and $R=1.615 \times 10^{-2} \mathrm{~cm}$ the radius of the beam. 


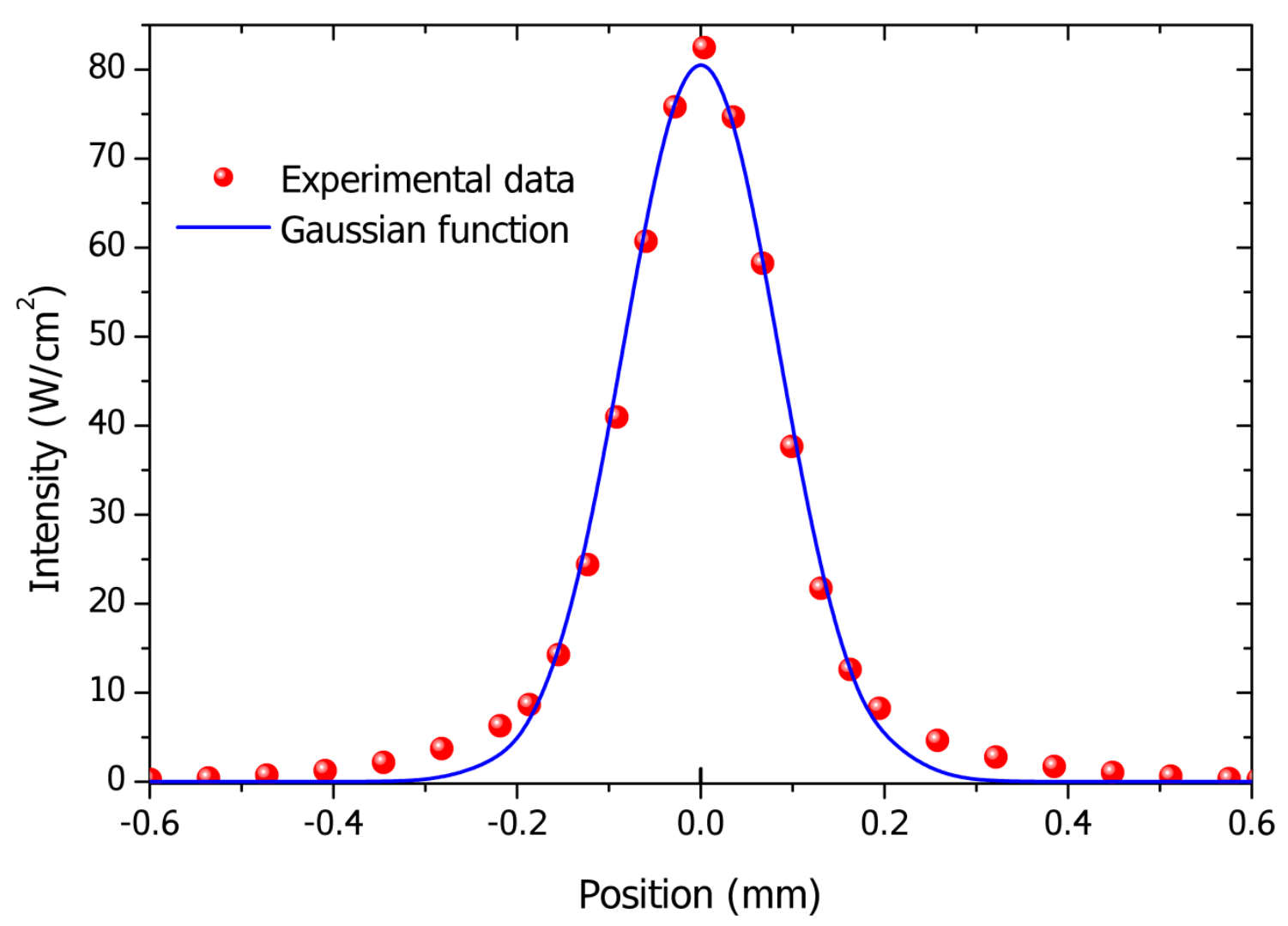

FIG 1: Intensity profiles of the laser beam, which is rotationally symmetrical. Red dots correspond to experimental laser beam profile and the blue line corresponds to Gaussian fit using Eq. (2).

The saturation level of the eigenstrain corresponding to photoexpansion is then taken to be proportional to the incident intensity and the photoexpansion kinetic is characterized by a single time parameter, independent of the incident intensity. For the sake of simplicity, we adopt the following uncoupled quasi-static photoexpansion evolution (see details in the Supplemental Information section):

$$
\epsilon^{p}(r, t)=\Lambda \cdot \exp \left[-\frac{2 \cdot r^{2}}{R^{2}}\right] \cdot\left(1-\exp \left[-\frac{t}{\tau_{A 0}}\right]\right)
$$

With $\tau_{A 0}$ the characteristic duration of photoexpansion and $\Lambda$ the asymptotic value of the "linear photoexpansion coefficient" at $I_{0}$. If a stress-free sample of thickness $e$ is irradiated by a homogenous light of intensity $I$, it will expand at $t \rightarrow \infty$ by $\Delta e$, so that:

$$
\Lambda=\frac{\Delta e}{e} \cdot \frac{I}{I_{0}}
$$

\subsection{Shear relaxation time}

It has been previously demonstrated that the shear relaxation time $\tau_{0}$ under bang gap irradiation is inversely proportional to the density of photons absorbed per second per atom [30]. Under 
irradiation, photoinduced entropy changes occur and it can impact the viscosity $\eta$. The viscosity is proportional to the shear relaxation time according to the Maxwell's relationship: $\eta=\tau_{0} \mu$. In order to simplify the model, we will only consider the average shear relaxation time of an irradiated volume, corresponding to a cylinder of thickness $e$ and of laser beam radius $R$. The shear relaxation time, for $r>R$ in the polar coordinate system $(r ; z)$ outside the laser beam is then set at $\tau_{0} \rightarrow \infty$, since for the $\mathrm{GeSe}_{9}$ glass, in the dark at configurational equilibrium, it is supposed to be larger than centuries [30]. Nevertheless, to insure the linearity of the proposed simple constitutive equations, the same relaxation time is assumed everywhere. This assumption implies that the glass is considered to be "photofluid" outside the irradiated volume, but this artifact has no practical consequence since the stress outside the laser beam is negligible, so that the impact of the relaxation time becomes negligible as well. The average shear relaxation time $\tau_{0}$, regarding the irradiated volume, is:

$$
\tau_{0}=\frac{N_{d}}{n} \cdot \pi \cdot R^{2} \cdot e \cdot\left[(1-\zeta) \cdot P \cdot(1-\exp [-\alpha \cdot e]) \cdot \frac{\lambda}{h c}\right]^{-1}=257.1 \mathrm{~s}
$$

where $N_{d}$ is the number of atoms per unit volume, $\zeta$ the reflectance at the considered wavelength, $P$ the power of the laser at the surface of the sample, $\alpha$ the absorption coefficient, $\frac{h c}{\lambda}$ the energy of a photon, and $n$ a proportionality factor equal to 0.2 for the $\mathrm{GeSe}_{9}$ glass [30]. The absorption coefficient, at $790 \mathrm{~nm}$, takes into account the photodarkening [30]. The values of all the parameters used are detailed in Table I. The right term of Eq. (5), between parentheses, is the number of photons absorbed per second. The term left of the parenthesis is the number of atoms in the irradiated volume divided by an efficiency parameter $n$.

TABLE I. Parameters used to calculate the shear relaxation time.

\begin{tabular}{ccc}
\hline Parameter & Description & Value \\
\hline $\mathrm{N}_{\mathrm{d}}$ & number of atoms per unit volume & $3.34 \times 10^{22} \mathrm{~cm}^{-3}($ ref. 30) \\
$\zeta$ & reflectance & 0.215 (ref. 30) \\
$e$ & sample thickness & $1.5 \mathrm{~mm}$ \\
$\lambda$ & laser wavelength & $790 \mathrm{~nm}$ \\
$\mathrm{hc} / \lambda$ & energy of a photon & $1.57 \mathrm{eV}$ or $2.51 \times 10^{-19} \mathrm{~J}$ \\
$R$ & beam radius & $1.615 \mathrm{~mm}$ \\
$P$ & power of the laser & $33.8 \mathrm{~mW}$ \\
$\alpha$ & absorption coefficient & $9.89 \mathrm{~cm}^{-1}(\mathrm{ref} .30)$ \\
\hline
\end{tabular}




\section{RESULTS}

\subsection{Analytical solution}

Instead of computing a rather difficult analytical solution based on use of potentials functions [36] (see for example an analogy with ref.[37] in the framework of thermoviscoelastic heated fibers embedded in a matrix), we have used the Laplace transform relative to the time $(t \rightarrow \mathrm{s})$ of our governing equations and we have constructed an approximated analytical solution with a form based on the separation of variables $r$ and $s$. This approach permits to simply return to the rt original space by inverse Laplace transform. The following approximated analytical expression for the vertical displacement is then obtained:

$$
u_{z}(r, z, t)=2 \cdot \Lambda \cdot \mathrm{z} \cdot \exp \left[-\frac{2 \cdot r^{2}}{R^{2}}\right] \cdot(1+f(t))
$$

where:

$$
f(t)=\frac{(1+v) \cdot\left(1-\frac{\tau_{A 0}}{\tau_{0}}\right) \cdot \exp \left[-\frac{t}{\tau_{A 0}}\right]+(1-2 v) \cdot \exp \left[\frac{(1+v)}{(2-v) \tau_{0}} \cdot t\right]}{(1+v) \cdot \frac{\tau_{A 0}}{\tau_{0}}+v-2}
$$

As expected, the vertical displacement reaches an asymptotic value at $t \rightarrow \infty$, since $\lim _{t \rightarrow \infty} f(t)=0$. This displacement is both proportional to the light intensity and to $z$. The maximum of the vertical displacement is obtained at the sample surface corresponding to the center of the beam: $u_{z}(0, e / 2, \mathrm{t})$ where the laser intensity is maximum.

\subsection{Fitting parameters}

From Eq. (6), we obtain the maximum displacement after extended irradiation time according to:

$$
\lim _{t \rightarrow \infty} f(t)=0
$$

Eq. (8) can then be fitted to the plateau of the experimental points plotted on Figure 2 to yield $\Lambda \sim 3.42 \times 10^{-3}$. We implicitly assume here that the eigenstrain $\epsilon^{\mathrm{p}}$ does not disappear instantaneously (even partially) since the experimental points correspond to measurements postirradiation and not under irradiation. Using $\tau_{\mathrm{A} 0} \sim 257.1 \mathrm{~s}$, as calculated from Eq. (5), we obtain, by fitting all the experimental points: $\tau_{\mathrm{A} 0} \sim 267.5 \mathrm{~s}$. Compared to the vertical displacement and the expansion radius, the radial displacement is found to be rather negligible and has little influence on the widening of the expansion profile. The maximum radial displacement at $t=$ $2400 \mathrm{~s}$ is located at the surface at $r=0.125 \mathrm{~mm}$, and reaches only $0.125 \times 10^{-3} \mathrm{~mm}$, whereas $u_{z}=$ $1.55 \times 10^{-3} \mathrm{~mm}$ at the same position and time. 


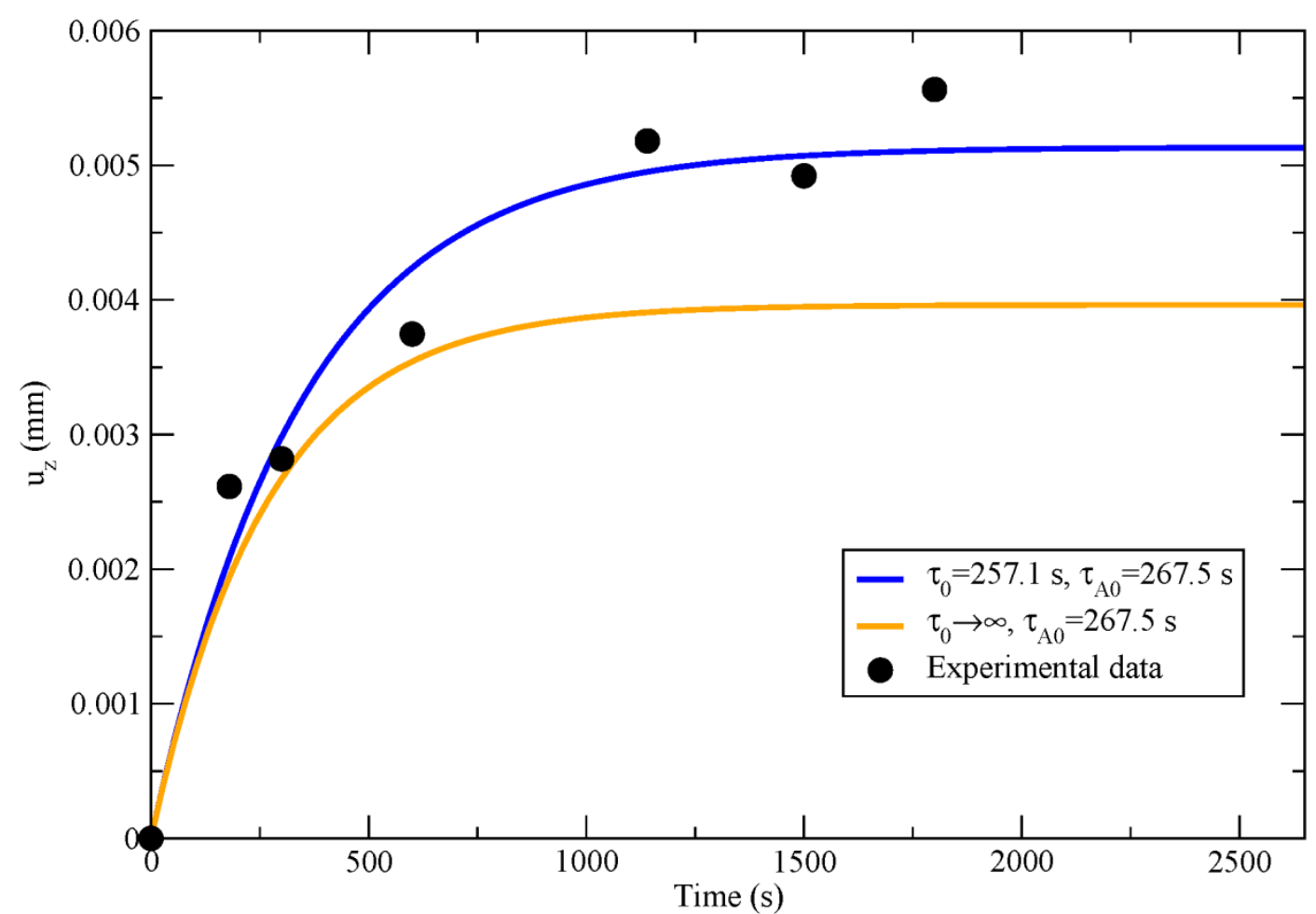

FIG. 2. Experimental vertical displacement of the surface, at $\mathrm{r}=0: u_{z}(0, e / 2, \mathrm{t})$ (dots); and displacement predicted by Eq. (6) (lines) at the same position, when taking into account the contribution of photofluidty with a relaxation time of $\tau_{0}=257.1 \mathrm{~s}$ derived from Eq. (5): top curve; and when assuming no contribution of photofluidity $\tau_{0} \rightarrow \infty$,: bottom curve.

\subsection{Comparison with experimental results}

Figure 2 shows a comparison of the experimental maximum expansion with the theoretical one obtained from our model. We first observe that the experimental data are affected by a significant uncertainty illustrated by the oscillation around the saturation level of photoexpansion. We must recall that the measurements are not performed in situ but instead each data point corresponds to a separate expansion performed on distinct points on the sample. This range of experimental uncertainty is observed in the data of other authors [14]. Ideally, measurements should be performed in-situ to optimally match model values. Nevertheless, the experimental data clearly show that the expansion takes place continuously, which supports the assumption that the eigenstrain $\epsilon^{\mathrm{p}}$ is time-dependent. Figure 2 also illustrates that if the photofluidity is not taken into account, i.e. if $\tau_{0} \rightarrow \infty$, the photoexpansion is considerably lower. This clearly illustrates the role of the photofluidity. Figure 3 shows a comparison of the experimental profiles with those predicted by our model. We can see that at $r<R$, the experimental profiles are nearly Gaussian, and that the radius of this Gaussian shape remains almost unchanged over time, and is close to $R$. This is consistent with the negligible radial displacement found in our calculation. Nevertheless, the experimental profiles deviate from the Gaussian shape as soon as $r>R$, even at short times, and this is not predicted by our 
calculations. This can be explained in part by the fact that the experimental beam is not perfectly Gaussian as shown in Fig. 1. This could also be associated with some contribution of the beam reflection on the convex surface within the sample. Both experimental data and calculation lead to a saturation of the photoexpansion at long times. The impact of the assumptions used in our model with regard to the small differences between experimental and theoretical profiles are discussed in the next section.

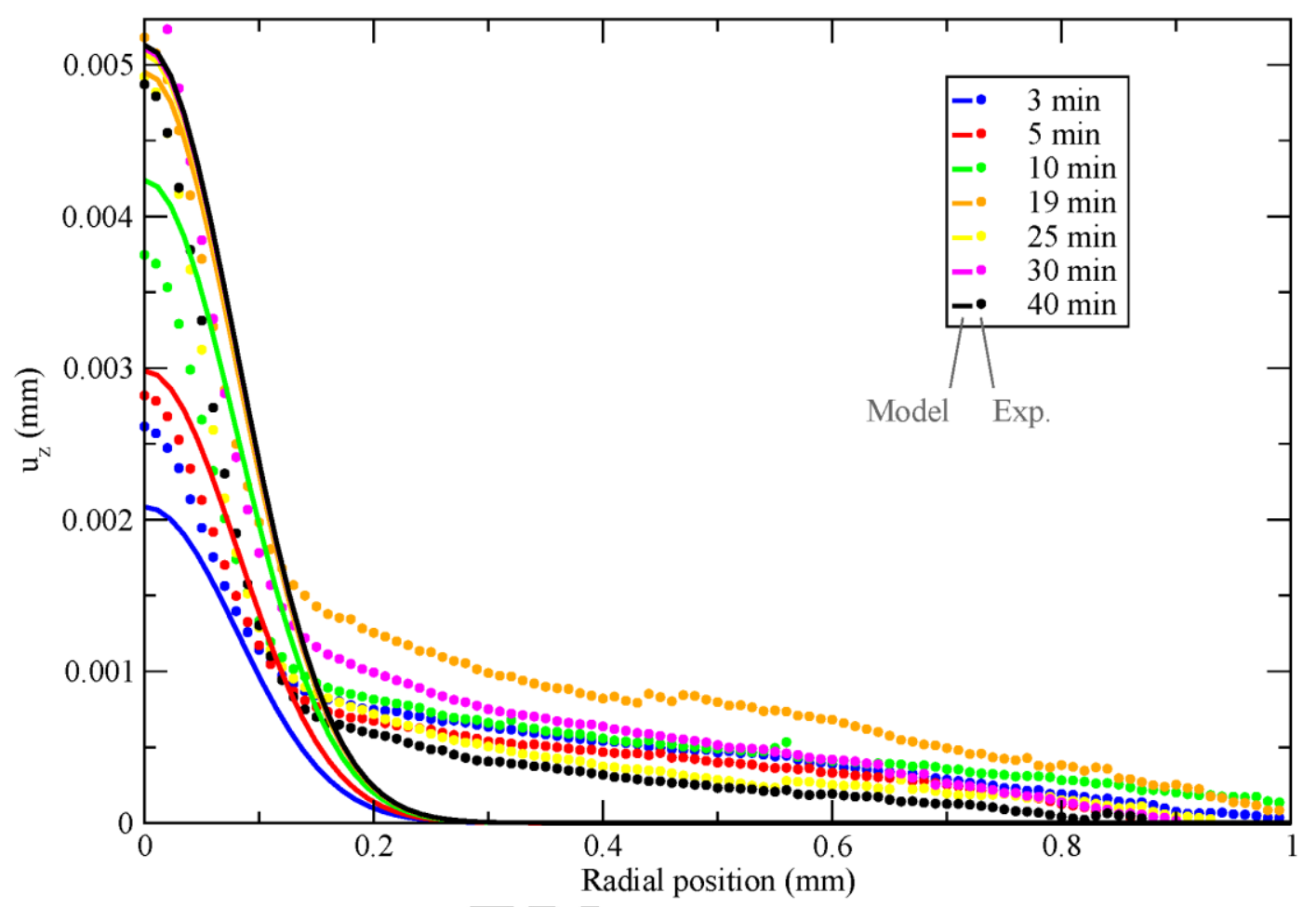

FIG. 3. Expansion profiles at various times of exposure: experiments (dots) and model (lines).

\section{DISCUSSION}

\subsection{Thermal contribution}

Recently, Zhao et al. [21] have suggested that thermal contributions to the giant photoexpansion may not be negligible. They have treated the photoexpansion using a thermoelastic approach, in the framework of uncoupled thermoelasticity, using the Beer's law to estimate the heat. This approach clearly presumes that even with low absorption, there is a nonnegligible heat production leading to thermal expansion. However, it does not take into account the photofluidity, and, as noted by the authors, thermal expansion cannot be the sole contribution since it cannot explain the photocontraction phenomenon. Another interpretation proposed by Calvez et al. [15], suggests instead that the main contributors to photocontraction and photoexpansion are photorelaxation and photoexcitation respectively.

Our model does not take into account the thermal contribution described by Zhao et al. [21] since we assume negligible light absorption. However, we can calculate the maximum 
temperature increase during our experiment using the model proposed by Zhao et al. Using the data detailed in Table II [13, 30, 33, 38], we obtain $\Delta T \sim 33.7 \mathrm{~K}$ at $t=2400 \mathrm{~s}$.

TABLE II. Necessary parameters in the thermoelastic model of Zhao et al.(ref.21), at $20^{\circ} \mathrm{C}$.

\begin{tabular}{ccc}
\hline Parameter & Description & Value \\
\hline$D$ & Thermal diffusivity & $3.22 \times 10^{-3} \mathrm{~cm}^{2} \cdot \mathrm{s}^{-1}$ (ref. 42) \\
$C_{p}$ & heat capacity & $\sim 0.3 \mathrm{~J} \cdot \mathrm{g}^{-1} \cdot \mathrm{K}^{-1}$ (ref. 13) \\
$\rho$ & density & $4.34 \mathrm{~g} \cdot \mathrm{cm}^{-1}$ (ref. 36) \\
$\alpha T$ & thermal expansion coefficient & $380 \times 10^{-7} \mathrm{~K}^{-1}$ (ref. 30) \\
\hline
\end{tabular}

Based on their thermoelastic model, we can evaluate the resulting maximum expansion: $u_{z}(\mathrm{t}=$ $2400) \sim 2.9 \times 10^{-3} \mathrm{~mm}$. By comparison to the maximum expansion of $\sim 5 \times 10^{-3} \mathrm{~mm}$ observed experimentally, this would indicate that, the thermoelastic expansion appears to be the major contribution. But the model of Zhao et al. predicts a broadly distorted expansion profile which is not in agreement with experiment as depicted on Fig. 4.

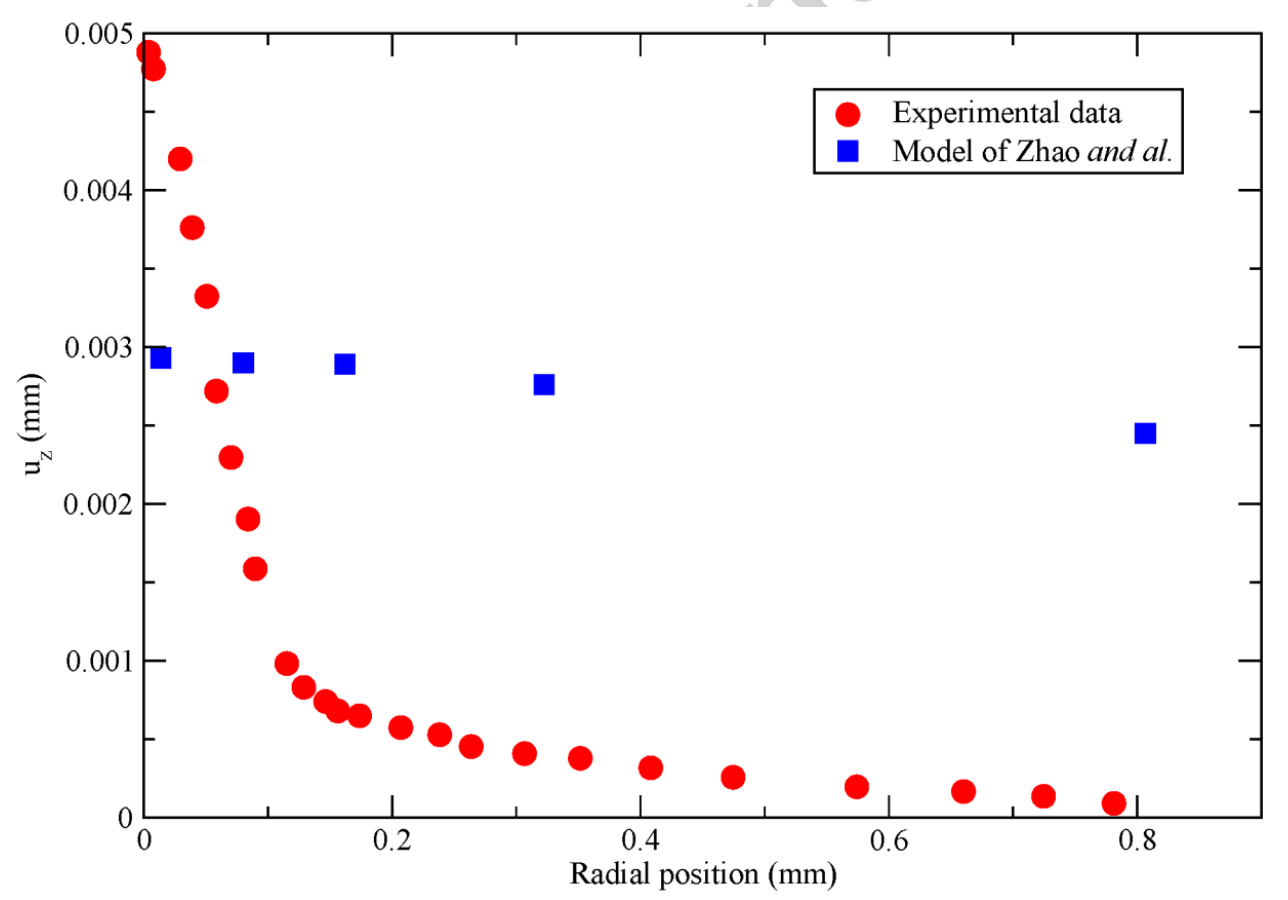

FIG. 4. Experimental expansion pro_le at $\mathrm{t}=2400 \mathrm{~s}$ and expansion predicted by the model of Zhao et al. (ref.21) at this time.

The model of Zhao et al. largely overestimates the expansion diameter in comparison to experimental observation. More specifically, the expansion diameter is notably larger than the beam diameter. This is not consistent with giant photoexpansion experiments where the diameter 
of the expansion is typically the same as the diameter of the laser beam according to various experimental reports $[14,22]$. Similarly, Zhao et al. find theoretical expansion diameters at least 2 order of magnitude larger[21] when modeling the experiment of Hisakuni \& Tanaka. Hence, the heat generated during photoexpansion seems to be largely overestimated in the model of Zhao et al., probably because photon energy is not mainly transferred into phonons, but rather contributes to the athermal eigenstrain of photoexpansion.

In order to investigate the extent of heat production during our photoexpansion experiment, we have performed a thermal imaging measurement derived from ref.[39]. Figure 5(a) shows a thermal image of a bulk $\mathrm{GeSe}_{9}$ sample irradiated with a laser intensity of $4.24 \mathrm{~W} \cdot \mathrm{cm}^{-2}$, at 785 $\mathrm{nm}$, placed in front of a heat/cool plate stabilized at $40^{\circ} \mathrm{C}$. The plate provides an upper temperature reference for the image. For comparison a $\mathrm{GeSe}_{9}$ disk heated at $40^{\circ} \mathrm{C}$ is shown next to a similar disk at room temperature in Fig. 5(c). It is clear from these two figures that the irradiated sample does not even reach $40^{\circ} \mathrm{C}$, since it still contrasts with the plate. Nevertheless, Fig. 5(b) clearly highlights that an expansion occurs, with a diameter of $1.5 \mathrm{~mm}$, corresponding to the laser beam diameter. According to the model of Zhao et al., the temperature increase should exceed $100^{\circ} \mathrm{C}$ in the middle of the expansion and $40^{\circ} \mathrm{C}$ at its border, after less than $3 \mathrm{~s}$. We have done this calculation using an absorption coefficient of $42 \mathrm{~cm}^{-1}$ at $785 \mathrm{~nm}$, taking into account the photodarkening [30]. This clearly illustrates that the heat is largely overestimated, or more precisely, that most of photon energy is not transferred into phonons but mainly converted in the creation of eigenstrain associated to photoexpansion.

Nevertheless, it must be pointed out that the experimental expansion profiles are not exactly Gaussian, but instead show a slight expansion at values larger than the beam diameter $r>R$. As shown above, this profile is not consistent with heat production due to absorption but may be partly explained by the fact that the experimental beam is not perfectly Gaussian (Fig. 1), as well as the possible contribution of reflections on the back surface of the glass sample. These back reflections could be significant due to the high refractive index of chalcogenide glasses and may not be coaxial with the incident beam due to self-focusing or deformation of the back surface as shown on Figure 5(b). 

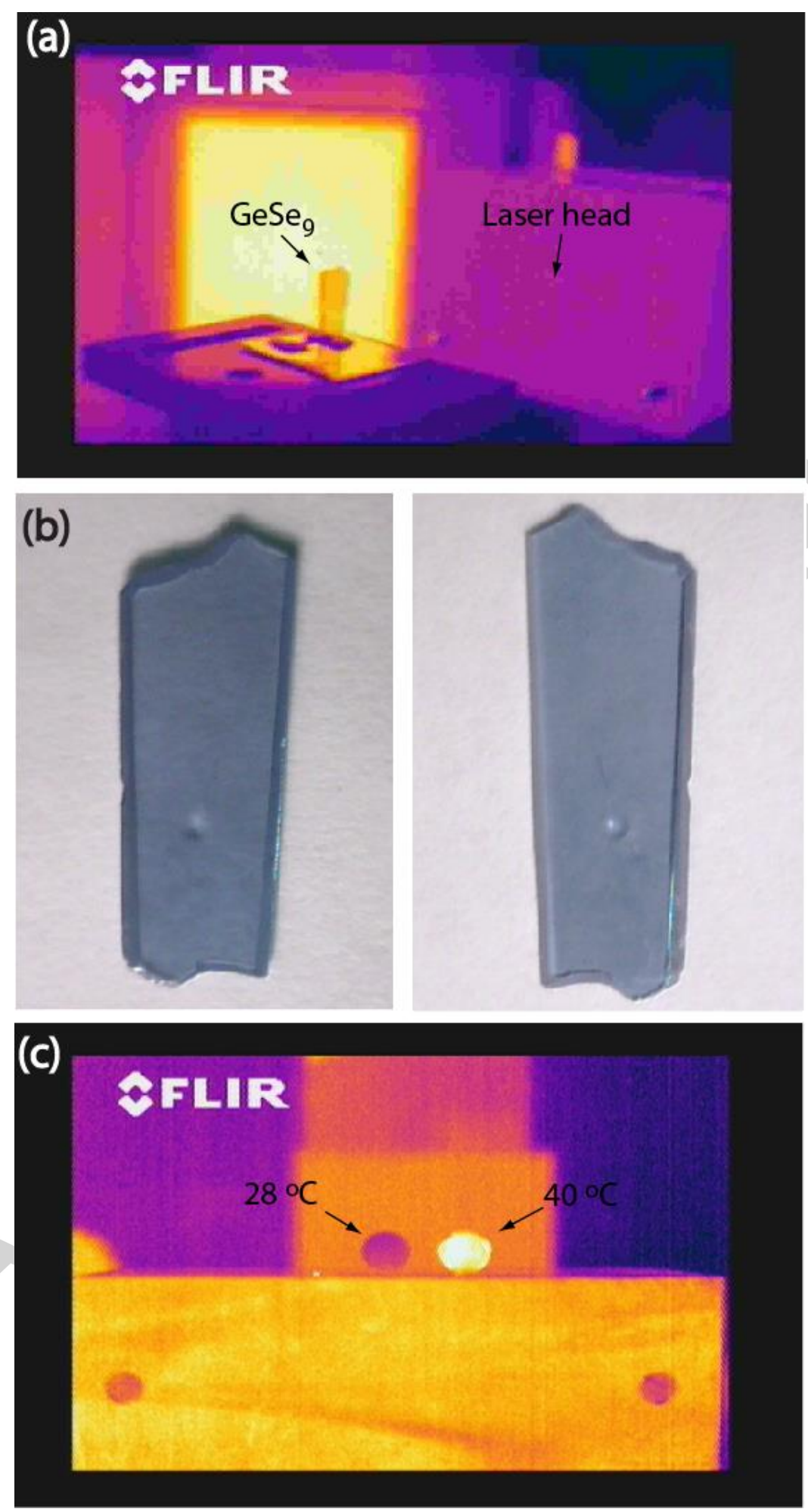

FIG. 5. (a) Thermograph of a bulk $\mathrm{GeSe}_{9}$ glass irradiated with a laser beam of intensity $150 \mathrm{~mW}$ and wavelength $785 \mathrm{~nm}$. The thermogram was collected with a FLIR Thermocam E300 IR camera. The background behind the sample corresponds to a heat/cool plate Echotherm IC120 tuned at exactly 40C. (b) The same bulk sample after an irradiation period, with a giant photoexpansion of diameter $1.5 \mathrm{~mm}$, on each side. (c) Thermograph of two bulk $\mathrm{GeSe}_{9}$, at $28^{\circ} \mathrm{C}$ on the left and $40^{\circ} \mathrm{C}$ on the right. 


\subsection{The role of photofluidity}

\subsection{1 . Does photofluidity enhance photoexpansion?}

It has been suggested, first by Tanaka et al. [27], that the photofluidity enhances the giant photoexpansion. Nevertheless, up to now, no model existed to demonstrate the role of the photofluidity. We can compare here the maximum expansion, $\lim _{t \rightarrow \infty} u_{z}(0, z, t)$, produced without photofluidity when $\tau_{0} \rightarrow \infty$, and the expansion produced with photofluidity, when $\tau_{0}$ is finite and positive.

$$
\frac{\lim _{t \rightarrow \infty} u_{z}(0, z, t)}{\lim _{t \rightarrow \infty}\left(\lim _{\tau_{0} \rightarrow \infty} u_{z}(0, z, t)\right)}=\frac{2-v}{1+v}
$$

This ratio is larger than unity and is a decreasing function of $v$ whenever $v \in[0 ; 1 / 2[$ : the maximum relative enhancement of photo expansion due to photofluidity occurs when the Poisson's ratio is null and corresponds to a value of $100 \%$. For the $\mathrm{GeSe}_{9}$ glass, the photofluidity enhances the relative expansion by almost 30\%. Eq. (9) shows that the contribution of photofluidity enhances the effect of an existing $\epsilon^{\mathrm{p}}$; however, due to other existing contributions and material properties, Eq. (9) is not sufficient to consider that the giant expansion is larger for materials with lower Poisson's ratio.

\subsubsection{Simplifying assumption considering the viscous flow}

In order to obtain an analytical solution, we have set $\tau_{0}(r)=\tau_{0}$. This corresponds to the assumption that the whole glass becomes photofluid, even outside the irradiated volume, at $r>R$. We have underestimated the shear relaxation time at $r>R$, so we have overestimated the viscous strain and consequently the vertical displacement at $r>R$. Nevertheless, the vertical displacement is proportional to the local laser intensity, and thus is negligible at $r>R$, even if it is overestimated. Indeed, since the stress is very low far from the middle of the laser beam, the underestimation of the shear relaxation time does not really impact the viscous strain in this region.

\subsubsection{Photofluidity and deviatoric stress}

In our model, the viscous flow is only produced by deviatoric stress, and not affected by hydrostatic pressure. This hypothesis comes from the comparison of the tensile and shear relaxation functions of Ge-Se glass fibers under irradiation [32] which shows that the viscous flow tends to become volume-conservative. This is a common hypothesis, indeed the Trouton law which states that the "coefficient of viscosity is equal to one third of the coefficient of viscous traction", derives from the same hypothesis. Hence, we can assume that if the hydrostatic pressure plays a role in the viscous flow, it is minor. According to this hypothesis, the viscous flow does not allow the relaxation of the hydrostatic pressure, even at long time and even when the irradiation ceases. Thus, the expansion stays stressed until the "natural" structural relaxation produces a volume relaxation that decreases the residual stress. 


\subsection{Photocontraction}

In our model, the eigenstrain due to photoexpansion is proportional to $\Lambda$. If we assume that the volume expansion is correlated to a photoinduced entropy increase, the opposite effect, the photoinduced relaxation, will promote a volume relaxation and a giant photocontraction [15]. We can take into account this phenomenon by changing the sign of $\Lambda: \Lambda>0$ corresponds to photoexpansion, and $\Lambda<0$ to photocontraction. In this latter situation, we can see from Eq. (6), that the photofluidity will also enhance the contraction.

\section{CONCLUSION}

It has been previously suggested that photofluidity plays a role in the giant photoexpansion effect, but so far no model has been proposed to quantify its contribution. The mechanical model in this study demonstrates for the first time that photofluidity indeed plays a major role in the giant photoexpansion process and establishes a numerical framework to quantify its contribution. In this model the photoexpansion is described as a new kind of athermal eigenstrain that induces local stress fields called egeinstress. The viscoelastic behavior generated by the photofluidity in turn enables these stresses to produce a giant photoexpansion. With some simple hypothesis, especially regarding the relationship between the eigenstrain and the light intensity, the problem of giant photoexpansion can be analytically solved and it is shown to provide a good prediction of experimental data both in term of magnitude and geometry of expansion. In addition, the model permits to emphasize the coherence between the viscosity under irradiation and the kinetic of giant photoexpansion. This model supports the idea that the photofluidity plays an important role in the giant photoexpansion in terms of amplitude and kinetic but not as important as expected by Tanaka [27] concerning the amplitude. More advanced analytical solutions will be required to treat complex optical effects such refractive index changes or self-focusing where the relationship between the light intensity and the eigenstrain is refined or where the light intensity profile is more sophisticated (for specific relief patterning, as an example). Nevertheless, the proposed model can serve as a starting point to implement the proposed integration law into more advanced numerical simulation that take more complex physical effects into account.

\section{ACKNOWLEDGEMENT}

The authors would like to thank the CNRS for supporting our international collaboration via the program PICS PhotoGlass (PICS 118254). P. L. acknowledges funding from the National Science Foundation (NSF Grant ECCS-1201865). M. B. acknowledges support from "La Fondation Rennes1, (semestre innovation)". 


\section{REFERENCES}

[1] J.L. Adam, X.H. Zhang, Chalcogenide Glasses, Woodhead Publishing Ltd., 2014.

[2] B.J. Eggleton, B. Luther-Davies, K. Richardson, Chalcogenide photonics, Nat. Photonics, 5 (2011) $141-148$.

[3] X.H. Zhang, Y. Guimond, Y. Bellec, Production of complex chalcogenide glass optics by molding for thermal imaging, J. Non-Cryst. Solids, 326\&327 (2003) 519-523.

[4] J. Troles, Q. Coulombier, G. Canat, M. Duhant, W. Renard, P. Toupin, L. Calvez, G. Renversez, F. Smektala, M. El Amraoui, J.L. Adam, T. Chartier, D. Mechin, L. Brilland, Low loss microstructured chalcogenide fibers for large non linear effects at 1995 nm, Opt. Express, 18 (2010) 26647-26654.

[5] P. Lucas, M.R. Riley, C. Boussard-Pledel, B. Bureau, Advances in chalcogenide fiber evanescent wave biochemical sensing, Anal. Biochem., 351 (2006) 1-10.

[6] M. Wuttig, N. Yamada, Phase-change materials for rewriteable data storage, Nat. Mater., 6 (2007) 824-832.

[7] A. V. Kolobov, K. Tanaka, Photoinduced phenomena in amorphous chalcogenides: from phenomenology to nanoscale, in: H.S. Nalwa (Ed.) Handbook of Advanced Electronic and Photonic Materials and Devices, Academic Press, New York, 2001, pp. 47.

[8] K. Shimakawa, A. Kolobov, S.R. Elliott, Photoinduced effects and metastability in amorphous semiconductors and insulators, Adv. Phys., 44 (1995) 475-588.

[9] J. Ren, T. Wagner, J. Orava, T. Kohoutek, B. Frumarova, M. Frumar, G.Yang, G. Chen, D. Zhao, A. Ganjoo, H. Jain, In-situ measurement of reversible photodarkening in ion-conducting chalcohalide glass, Opt. Express, 16 (2008) 1466-1474.

[10] H. Hisakuni, K. Tanaka, Optical microfabrication of chalcogenide glasses, Science 270 (1995) 974975.

[11] K. Tanaka, Photoinduced fluidity in chalcogenide glasses, C. R. Chim., 5 (2002) 805-811.

[12] R.Y. Golovchak, A. Kozdras, O.I. Shpotyuk, Physical ageing in glassy As-Se induced by above-band gap photoexposure, Solid State Commun., 145 (2008) 423-426.

[13] P. Lucas, A. Doraiswamy, E.A. King, Photoinduced structural relaxation in chalcogenide glasses, J. Non-Cryst. Solids, 332 (2003) 35-42.

[14] H. Hisakuni, K. Tanaka, Giant photoexpansion in As2S3 glass, Appl. Phys. Lett., 65 (1994) 29252927.

[15] L. Calvez, Z. Yang, P. Lucas, Reversible giant photocontraction in chalcogenide glass, Opt Express, 17 (2009) 18581-18589.

[16] P. Krecmer, A.M. Moulin, R.J. Stephenson, T. Rayment, M.E. Welland, S.R. Elliott, Reversible nanocontraction and dilatation in a solid induced by polarized light, Science 277 (1997) 1799-1802.

[17] A.V. Kolobov, Photo-induced metastability in amorphous semiconductors, in, WILEY-VCH GmbH \& Co. KGaA, Weinheim, 2003.

[18] A. Kozdras, R. Golovchak, O. Shpotyuk, S. Szymura, A. Saiter, J.-M. Saiter, Light-assisted physical aging in chalcogenide glasses: dependence on the wavelength of incident photons, J. Mater. Res., 26 (2011) 2420-2427.

[19] K. E. Asatryan, T. Galstian, R. Vallee, Optical polarization driven giant relief modulation in amorphous chalcogenide glasses, Phys. Rev. Lett., 94 (2005) 087401.

[20] K. Tanaka, N. Toyosawa, H. Hisakuni, Photoinduced Bragg gratings in As2S3 optical fibers, Opt. Lett., 20 (1995) 1976-1978.

[21] D. Zhao, H. Jain, L.C. Malacarne, P.R.B. Pedreira, Role of photothermal effect in photoexpansion of chalcogenide glasses, Phys. Status Solidi B, 250 (2013) 983-987. 
[22] A. Saitoh, K. Tanaka, Self-developing aspherical chalcogenide-glass microlenses for semiconductor lasers, Appl. Phys. Lett., 83 (2003) 1725-1727.

[23] H. Hisakuni, K. Tanaka, Optical fabrication of microlenses in chalcogenide glasses, Opt. Lett., 20 (1995) 958-960.

[24] A. Saitoh, T. Gotoh, K. Tanaka, Chalcogenide-glass microlenses for optical fibers, J. Non-Cryst. Solids, 299-302 (2002) 983-987.

[25] S. H. Messaddeq, V. K. Tikhomirov, Y. Messaddeq, D. Lezal, M.S. Liu, Light-induiced relief grating and a mechanism of metastable light-induced expansion in chalcogenide glasses, Phys. Rev. B, 63 (2001) 224203.

[26] Z. Yang, N.C. Anheier, Jr., H.A. Qiao, P. Lucas, Simultaneous microscopic measurements of photodarkening and photoexpansion in chalcogenide films, J. Phys. D: Appl. Phys., 42 (2009) 135412/135411-135412/135415.

[27] K. Tanaka, A. Saitoh, N. Terakado, Giant photo-expansion in chalcogenide glass, J. Optoelectron. Adv. Mater., 8 (2006) 2058-2065.

[28] R. Lukacs, S.D. Baranovskii, P. Thomas, F. Gebhard, To the kinetics of photoinduced volume changes in chalcogenide glasses, J. Appl. Phys., 103 (2008) 093541/093541-093541/093544.

[29] T. Mura, Micromechanics of Defects in Solids, 2nd ed., Kluwer Academic Publishers, 1987.

[30] Y. Gueguen, J.C. Sangleboeuf, V. Keryvin, E. Lepine, Z. Yang, T. Rouxel, C. Point, B. Bureau, X.H. Zhang, P. Lucas, Photoinduced fluidity in chalcogenide glasses at low and high intensities: A model accounting for photon efficiency, Phys. Rev. B: Condens. Matter 82 (2010) 134114/134111-134112. [31] J. Li, D.A. Drabold, Direct calculation of light-induced structural change in diffusive motion in glassy $\mathrm{As}_{2} \mathrm{~S}_{3}$, Phys. Rev. Lett., 85 (2000) 2785.

[32] Y. Gueguen, Reologie de verres speciaux dans le domaine de transition et phenomenes photoinduits, in, Universie de Rennes 1, 2010.

[33] J.-P. Guin, T. Rouxel, V. Keryvin, J.-C. Sangleboeuf, I. Serre, J. Lucas, Indentation creep of Ge-Se chalcogenide glasses below Tg: elastic recovery and non-Newtonian flow, J. Non-Cryst. Solids, 298 (2002) 260-269.

[34] B. Halphen, Q. Nguyen, J. Mecanique, 14 (1975) 39.

[35] P. Lucas, E.A. King, Calorimetric characterization of photoinduced relaxation in GeSe9 glass, J. Appl. Phys., 100 (2006) 023502/023501-023502/023506.

[36] W. Nowacki, Thermoelasticity, 2nd ed., Pergamon, 1986.

[37] N.R. Sottos, R.L. McCullough, W.R. Scott, The influence of interphase regions on local thermal displacements in composites, Compos. Sci. Technol., 44 (1992) 319-332.

[38] A. Mathew, J. Ravi, K.N. Madhusoodanan, K.P.R. Nair, T.M.A. Rasheed, Thermal diffusivity measurements of semiconducting amorphous GexSe100-x thin films by photothermal deflection technique, Appl. Surf. Sci., 227 (2004) 410-415.

[39] E. Lepine, Z. Yang, Y. Gueguen, J. Troles, X.-H. Zhang, B. Bureau, C. Boussard-Pledel, J.-C. Sangleboeuf, P. Lucas, Optical microfabrication of tapers in low-loss chalcogenide fibers, J. Opt. Soc. Am. B, 27 (2010) 966-971. 